\section{DELETION OF THE BAFF RECEPTOR TACI FULLY PROTECTS AGAINST SLE WITHOUT REDUCTION OF B CELL NUMBERS AND FUNCTION}

${ }^{1} \mathrm{~F}$ Mackay*. ${ }^{1}$ University of Melbourne, School of Biomedical Sciences, University of Melbourne, Australia

\subsection{6/lupus-2017-000215.345}

Background and aims B cell-activating factor of the TNF family (BAFF) is an essential B cell survival factor. However, high levels of BAFF promote systemic lupus erythematosus (SLE) in mice and humans. Belimumab (anti-human BAFF) limits B cell survival and is approved for use in patients with SLE. Surprisingly, the efficacy of rituximab in SLE remains controversial, despite depleting $\mathrm{B}$ cells more potently than belimumab. This raises the question of whether $\mathrm{B}$ cell depletion is really the mechanism of action of belimumab. In BAFF transgenic (BAFF-Tg) mice, SLE development is $\mathrm{T}$ cell-independent but relies on innate activation of $\mathrm{B}$ cells in cooperation with the BAFF receptor TACI. Therefore, in this study we tested whether TACI, a BAFF receptor dispensable for B cell survival may have a role in the pathogenesis of SLE.

Methods To test the role of TACI in driving BAFF-mediated autoimmunity, we reconstituted BAFF $\mathrm{Tg}$ mice with a TACIdeficient bone marrow and also crossed BAFF $\mathrm{Tg}$ mice onto $\mathrm{TACI}^{--}$mice.

Results We show that loss of TACI on B cells protected against BAFF-mediated autoimmune manifestations while preserving B cells, suggesting that loss of BAFF signalling through TACI, rather than loss of $\mathrm{B}$ cells, may underpin the effect of belimumab in the clinic. Moreover, a multimeric form of BAFF, is very effective at activating TACI, suggesting that this abnormal form of BAFF may also be a pathogenic factor in SLE.

Conclusions B cell-sparing blockade of TACI may offer a more specific and safer therapeutic alternative to broad B cell depletion in SLE.

\section{THE CONTRIBUTION OF INTERFERON LAMBDA TO SLE}

${ }^{1} \mathrm{C}$ Macri ${ }^{*},{ }^{1} \mathrm{ES}$ Pang, ${ }^{1} \mathrm{~J}$ Pooley, ${ }^{2} \mathrm{~K}$ Radford, ${ }^{1} \mathrm{M}$ O'Keeffe. ${ }^{1}$ Monash University, Biochemistry and Molecular Biology, Clayton, Australia; ${ }^{2}$ Mater Research-UQ, TRI, Brisbane, Australia

\subsection{6/lupus-2017-000215.346}

Background and aims Interferon lambda (IFN- $\lambda$ ) is a novel type of interferon produced by dendritic cells (DC). Despite its binding to a different receptor, IFN- $\lambda$ shares functional similarities with type I IFN (IFN-I) by upregulating the expression of IFN-stimulated genes. The role of IFN- $\lambda$ in DC biology and in autoimmunity remains unknown.

- to identify the DC subsets producing IFN- $\lambda$.

- to investigate the role of IFN- $\lambda$ in DC functions.

- to investigate the role of IFN- $\lambda$ in SLE.

Methods

- Mouse and human DC subsets were stimulated ex vivo and the IFN- $\lambda$ expression was measured.

- The maturation and the capacity of DC to cross-prime T cells was compared in WT and IFN- $\lambda \mathrm{R}^{-/-}$mice. T cell cross-priming by human DCs was measured ex vivo in the presence of exogenous IFN- $\lambda$.
- Serum levels of IFN- $\lambda$ was measured in lupus-prone mice and in SLE patients. The phenotype of the blood DC subsets from SLE patients was also characterised.

Results

- Mouse plasmacytoid DC (pDC) and $\mathrm{CD}^{+}$DC highly secrete IFN- $\lambda$. In humans, the CD $141^{+}$DC are the major IFN- $\lambda$ producers.

- IFN- $\lambda$ enhances the capacities of mouse and human DCs to maturate and to cross-prime T cells.

- High serum levels of IFN- $\lambda$ were detected in lupus-prone mice and in some SLE patients. SLE patients display increased activation of the IFN-producing DC subsets: the pDCs (producing IFN-I) and the CD $141^{+}$DCs (producing IFN- $\lambda$ ).

Conclusions IFN- $\lambda$ is produced by some DC subsets and enhances their functions. Furthermore, IFN- $\lambda$ is expressed during SLE, suggesting a potential role of the cytokine in the aetiology of SLE.

\section{7 IMPACT OF CD200-FC ON DENDRITIC CELLS IN LUPUS- PRONE NZB/WF1 MICE}

W Mo*, Y Yin, X Zhang. Beijing peking union medical college hospital, rheumatology, Beijing, China

\subsection{6/lupus-2017-000215.347}

Background and aims Abnormal expression of CD200/ CD200R1 may contribute to the immunologic abnormalities in patients with systemic lupus erythematosus (SLE). This study aimed to assess the function of CD200/CD200R1 and impact of CD200-Fc on dendritic cells in lupus-prone NZB/WF1 mice.

Methods Female NZB/WF1 mice were treated with CD200-Fc or control for 4 weeks. Plasma samples were collected to measure autoantibody levels. The expression levels of CD200/ CD200R1 in peripheral blood mononuclear cells (PBMCs) and splenocytes were examined.

Results The percentage of CD200/CD200R1-positive cells in splenocytes from NZB/WF1 mice was lower than that of C57BL/6 mice $(p<0.05)$. The plasma level of anti-dsDNA was significantly higher in NZB/WF1 mice than C57BL/6 mice $(p<0.001)$. However, the anti-dsDNA levels decreased $(\mathrm{p}=0.047)$ after CD200-Fc treatment. Finally, CD200-Fc reduced the levels of IL-6 $(p=0.017)$ and IL-10 $(p=0.03)$ in the dendritic cell culture supernatant.

Conclusions The immunosuppressive CD200/CD200R1 signalling pathway might be involved in the immunopathology of NZB/WF1 mice; the present results merit further exploration of agents that can modulate the CD200/CD200FR1 pathway as a therapy for human lupus

\section{DECTIN-1 ON MONOCYTIC CELLS MEDIATES ABERRANT INNATE AND ADAPTIVE IMMUNE RESPONSES IN PATIENTS WITH SYSTEMIC LUPUS ERYTHEMATOSUS}

${ }^{1} \mathrm{MY}$ Mok*, ${ }^{2} \mathrm{KY}$ Lam, ${ }^{2} \mathrm{D}$ Luk, ${ }^{2} \mathrm{Y}$ Lo. ${ }^{1}$ City University of Hong Kong, Department of Biomedical Sciences, Hong Kong, Hong Kong S.A.R; ${ }^{2}$ University of Hong Kong, Department of Medicine, Hong Kong, Hong Kong S.A.R

10.1136/lupus-2017-000215.348 
Background and aims Dectin-1 is a c-type lectin like receptor that signals via syk and is involved in anti-fungal immunity. Dectin-1 was found to trigger experimental inflammatory arthritis, and likely play a role in the pathogenesis of some autoimmune diseases. This study aimed to examine dectin-1 expression and function of circulating CD14+ monocytes and monocyte-derived dendritic cells (MDDCs) in patients with systemic lupus erythematosus (SLE)

Methods SLE patients with active and inactive diseases and healthy subjects were recruited. Dectin-1 agonists including curdlan, zymosan and toll-like receptor agonists Pam3CSK4 (TLR2) and LPS (TLR4) were used to stimulate monocytes and/or MDDCs. Dectin-1, ROS and phosphorylated-syk (pSyk) were measured by flow cytometry. Cytokine profile was measured by and multi-bead immunoassay.

Results Dectin-1 expressing monocytes was significantly lower in active SLE patients compared to inactive patients and healthy controls. The absolute count of dectin-1 expressing monocytes correlated significantly and inversely with SLEDAI, anti-dsDNA antibody level and C4. Despite this, ROS production upon stimulation by dectin-1 agonists was comparable. Stimulation of dectin-1 led to activation and maturation of MDDCs. SLE MDDCs showed higher p-Syk activation compared to normal MDDCs upon dectin-1 stimulation. Curdlanstimulated MDDCs produced higher levels of IL-1 $\beta$, IL-23 and TNF- $\alpha$. Adding TLR2 agonist to curdlan, SLE MDDCs produced significantly higher level of IL-1 $\beta$ compared to normal MDDCs.

Conclusions Active SLE patients had significantly lower circulating dectin-1 expressing monocytes which produced comparable level of ROS compared to inactive patients and healthy subjects. Dectin-1 agonists led to significantly higher Th17 promoting cytokines upon co-stimulation with TLR2 in SLE MDDCs.

\section{TMEM173/STING IS CRUCIAL FOR LUPUS DEVELOPMENT IN FCGR2B-DEFICIENCY MICE}

${ }^{1}$ P Pisitkun* ${ }^{2} \mathrm{~A}$ Thim-Uam, ${ }^{3} \mathrm{M}$ Tansakul, ${ }^{2} \mathrm{~B}$ Wongprom, ${ }^{4} \mathrm{~A}$ Leelahavanichkul, ${ }^{5} \mathrm{~S}$ Paludan, ${ }^{2}$ T Pisitkun. ${ }^{1}$ Faculty of Medicine Ramathibodi Hospital, Department of Medicine- Division of Allergy- Immunology- and Rheumatology, Bangkok, Thailand; ${ }^{2}$ Faculty of MedicineChulalongkorn University, Chulalongkorn University Systems Biology Centre, Bangkok, Thailand; ${ }^{3}$ Faculty of Medicine Ramathibodi Hospital, Translational Medicine Program, Bangkok, Thailand; ${ }^{4}$ Faculty of Medicine- Chulalongkorn University, Department of Microbiology, Bangkok, Thailand; ${ }^{5}$ Aarhus University, Department of Biomedicine, Aarhus, Denmark

\subsection{6/lupus-2017-000215.349}

Background and aims Type I interferon is one of the most critical cytokines involving in lupus pathogenesis. The activation of endosomal nucleic acid sensors leading to type I IFN production empowers the lupus phenotypes in several mouse models. The signalling of cytosolic DNA sensor also induces type I IFN production and the role in lupus disease are not clear. Stimulator of interferon genes (Sting), also known as transmembrane protein 173 (TMEM173) and MPYS/MITA/ ERIS, is a cytosolic DNA sensor which recognised cyclic diGMP and subsequently stimulated type I interferon production. The Fcgr2b-deficient mice develop spontaneous lupus phenotypes which are splenomegaly, the presence of antinuclear antibodies (ANA) and fatal glomerulonephritis. The polymorphisms of FCGR $2 B$ associates with the increases of lupus susceptibility in human. The goal of the study is to identify the role of Sting in lupus mouse model.

Methods The Sting-deficient mice were bred with the Fcgr2bdeficient mice to create double deficient mice and control littermates. The mice were analysed for survival rate, autoantibodies production, severity of pathology, gene expression profiles, and immunophenotypes.

Results In the absence of Sting, the Fcgr2b-deficient mice survived longer and the level of ANA and anti-dsDNA antibody considerably reduced. The glomerulonephritis in the doubledeficient mice also ameliorated. The expression of interferon

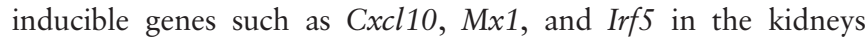
of the double-deficient mice was significantly lower than the Fcgr2b-deficient mice.

Conclusions Sting-mediated signalling pathway plays the substantial role in lupus pathogenesis of the Fcgr2b-deficient mice. Blocking Sting function may be the advantage for treatment in lupus patients.

\section{TOLL-LIKE RECEPTOR 7 SIGNALLING DRIVES TRANSITIONAL B CELLS EXPANSION AND AUTOANTIBODY PRODUCTION IN SYSTEMIC LUPUS ERYTHEMATOSUS}

${ }^{1} \mathrm{~T}$ Wang ${ }^{*},{ }^{2} \mathrm{~J}$ Marken, ${ }^{3} \mathrm{~K}$ Cerosaletti, ${ }^{1} \mathrm{M}$ Li, ${ }^{2} \mathrm{~K}$ Elkon, ${ }^{1} \mathrm{X}$ Zeng, ${ }^{2} \mathrm{~N}$ Giltiay. ${ }^{1}$ Peking Union Medical College Hospital, Rheumatology, Beijing, China; '2University of Washington, Rheumatology, Seattle, USA; ${ }^{3}$ Benaroya Research Institute at Virginia Mason, Translational Research Program, Seattle, USA

\subsection{6/lupus-2017-000215.350}

Background and aims Toll-like receptor 7 (TLR7) has been implicated in B cells activation and the generation of pathogenic autoantibodies. Newly-formed transitional (TR) B cells are enriched in autoreactive specificities and are increased in some SLE patients. This study was undertaken to examine a possible link between the TR B cells expansion/activation and TLR7 levels in SLE.

Methods PBMCs were collected from SLE patients and healthy donors and analysed for the expression of TLR7, TLR9 and IFN-responsive genes by RT-PCR. The frequencies of B cell populations were analysed by flow cytometry. BAFF titers were analysed by ELISA. TLR7 variant rs3853839(C/G) was detected by Taqman 5'-allele discrimination assay. TR B cells were primed with IFN $\alpha$ and stimulated with TLR7 ligands in vitro.

Results High expression levels of TLR7 in SLE patients positively correlated with IFN signature and disease activity, but not with BAFF titers. SLE patients with high levels of TLR7 (TLR7 ${ }^{\text {hi }}$ group) showed an expansion of CD $19^{+} \mathrm{CD} 38^{\text {high }} \mathrm{CD} 24^{\text {high }} \mathrm{CD} 10^{+}$TR B cells. Overall, frequencies of TR B cells positively correlated with the levels of TLR7, but not TLR9. SLE patients, carrying a risk G allele, had increased TLR7 expression and TR cell frequencies, compared to non-risk allele carriers. TLR $7^{\text {hi }}$ SLE patients showed increased autoantibody titers and skewing towards Sm/RNP antigens. Upon IFN $\alpha$ priming, TR B cells up-regulated TLR7 and differentiated into plasmablasts in response to TLR7ligand stimulation.

Conclusions Our findings suggest that dysregulation of TLR7 in SLE might drive the expansion and promote the activation of TR B cells, which might be a source of autoantibodies. 